\title{
Lessons from Ebola bring WHO reforms
}

$\mathrm{T}$ he World Health Organization is taking initial steps to reform its emergency response following criticism in a report by an independent panel of experts. That report analyzed what went wrong in the Ebola outbreak in West Africa and offered 21 recommendations to better prepare for a future outbreak.

Although progress has been made to enhance emergency response leadership, the challenge now is to implement an effective strategy for emergencies, and that hinges on funding and compliance by its 194 member states.

At a July 31 media conference, WHO (World Health Organization) Director-General Dr. Margaret Chan highlighted six recommendations that are being implemented to varying degrees. These include streamlining internal procedures to speed up emergency response; helping countries to create a resilient health system; establishing a global emergency workforce; encouraging countries to report outbreaks early; continuing research and development; and asking member states to contribute more financial support.

WHO has also created an advisory group of 19 experts led by Dr. David Nabarro, the United Nations SecretaryGeneral's Special Envoy on Ebola. The group will investigate the problems raised in the report and inform WHO how to respond to the remaining recommendations. "Culture change will be necessary," Nabarro says.

Some doctors and humanitarian organizations that worked in West Africa to stop the spread of the outbreak that infected about 27800 people and killed 11300 , see this as a positive start, but are concerned about potential weaknesses in the delivery of WHO's emergency response reforms.

Dr. Allison McGeer, a microbiologist and infectious disease consultant at Mount Sinai Hospital in Toronto, worked with WHO in Oct. 2014 and spent almost three months in Liberia. She says that this proposal is a step in

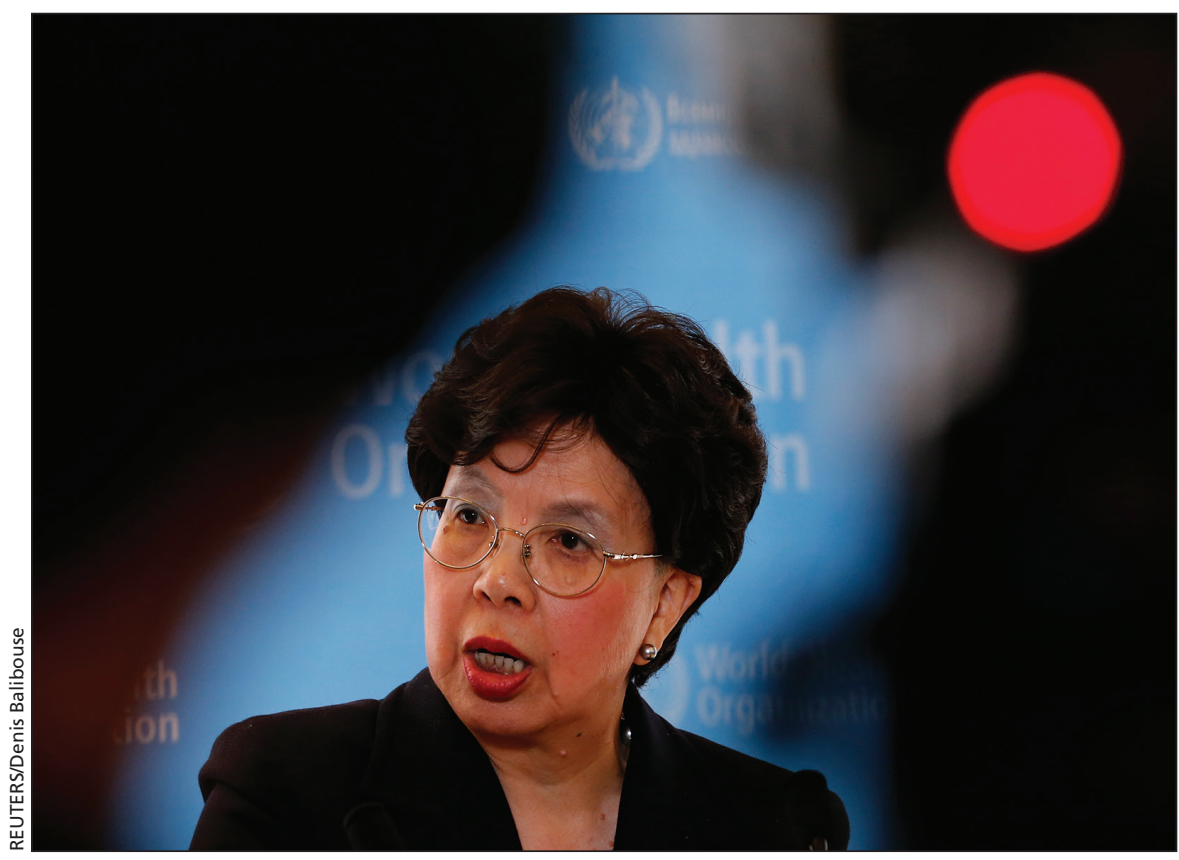

WHO Director-General Dr. Margaret Chan urges member nations to contribute to an emergency outbreak contingency fund; she did the same thing after the 2009 H1N1 outbreak, but to no avail.

the right direction, but "None of this is going to work unless member states are willing to step up and support it. We failed to do that before."

McGeer was referring to the 2011 report evaluating WHO's response to the $2009 \mathrm{H} 1 \mathrm{~N} 1$ (swine flu) pandemic in Mexico. Back then, it was recommended that member states contribute $\$ 100$ million to a contingency fund to fight future outbreaks; this never happened. This latest report recommends the same thing.

Dr. Kumanan Wilson, a senior scientist in the clinical epidemiology program at the Ottawa Hospital Research Institute, consulted for WHO on the International Health Regulations, a binding agreement with 196 countries on preventing public health crises. Measures to stop a potential spread are expensive, but "It's probably costeffective for wealthier countries to contribute to these funds to help low-tomiddle income countries develop the capacity to respond and $\mathrm{WHO}$ to have the capacity to assist them." The 2003 SARS outbreak cost Toronto \$1 billion.
So far only the United Kingdom has openly pledged $\$ 10$ million for the contingency fund; China and India have also pledged an undisclosed amount. When contacted, Canada's Department of Foreign Affairs refused to comment.

In its report, the independent panel also recommended creating incentives for countries to report outbreaks early, and disincentives for countries that don't follow the regulations, for example, by restricting travel.

Dr. Rob Fowler, a senior scientist at Sunnybrook Health Sciences Centre in Toronto, has been in West Africa periodically since March 2014. He says it's uncertain what will happen if countries don't follow the regulations. Canada contravened them by issuing a travel ban against people from West Africa without penalty. "There is no disincentive with teeth," Fowler says.

And that has far-reaching implications. "This is time when there is an increased need for communication and in-person meetings with people from West Africa, and when member states 
deny that opportunity, then you are denying optimal response and opportunities to control the outbreak."

Not all the recommendations require a coordinated response from member states. One of the reforms that is already showing promise is WHO's streamlined response mechanism, which has been applied to emergencies in Yemen, South Korea and Nepal. The Canadian Red Cross Director of Emergencies and Recovery, Hossam Elsharkawi, who was in Nepal after the earthquake, says WHO played a positive role with the Nepalese Ministry of Health in coordinating the many international medical teams as they arrived. "Between Ebola and Nepal, you could already see some progress."

Like many others, Elsharkawi expected WHO to take the lead during the Ebola crisis. He says the health systems in the three affected countries,
Guinea, Sierra Leone and Liberia, were weak to begin with and humanitarian organizations were missing key information about clinical protocols and treatments. "We seem to go on from one crisis or another and perhaps not applying the lessons."

\section{Canadian Ebola vaccine}

Although funding from member states remains unseen, WHO has shown its leadership role in a clinical study of Canada's VSV-EBOV Ebola vaccine. Early results published in The Lancet on July 31 suggest that the vaccine was $100 \%$ effective.

"Vaccines take years to develop. Nobody has ever done a vaccine trial during an outbreak before. The sheer logistical and ethical and organizational issues to do that are immense, yet people managed to pull it off," McGeer says.
The vaccine used in the trials in Guinea emulated the same "ring vaccination" approach used to eradicate smallpox in the 1970s, which tracked down and vaccinated anyone exposed to a patient with the infection. In the Ebola trials, some people were vaccinated right away and others were vaccinated 10 days later. Among the 2380 people who were vaccinated later, 16 developed Ebola; none of the 2014 people who received the vaccine immediately developed the disease.

The vaccine was developed in the Public Health Agency of Canada's National Microbiology Laboratory. The Canadian government then donated the experimental vaccine to WHO for further study; NewLink Genetics and Merck acquired the licensing rights. Shannon Lough, CMAJ

CMAJ 2015. DOI:10.1503/cmaj.109-5125 